\title{
Development of a Compact Container Protecting from Accidental Explosions of High Energy Materials
}

\author{
Teito Matsuishi ${ }^{1,{ }^{*}}$, Kazuhito Fujiwara ${ }^{1}$, Fumiko Kawashima ${ }^{1}$, Haruka Oda ${ }^{1}$ \\ ${ }^{1}$ Kumamoto University, Chuo-Ku, Kurokami 2-39-1, Kumamoto, 860-8555 Japan \\ *180d8476@st.kumamoto-u.ac.jp
}

Keywords: Ultra High Molecular Weight Polyethylene, Dyneema, Zylon, Compact Container, Suppressing Deformation

\begin{abstract}
There are many things in our surroundings that are at risk of explosion (e.g. lithium ion batteries, power modules, spray cans etc.), and there is a possibility of causing great damage to the surroundings due to explosive fragments. For safe operation of them, it is essential to establish a way to protect the surroundings from explosive fragments. In this study, the purpose is to develop a compact container protecting the surroundings from explosive fragments. UltraHigh Molecular Weight polyethylene (UHMW) which is excellent in strength against explosive and penetrationof fragments was used for container, and Dyneema string or Zylon string were wound around the container for suppressing deformation. In order to observe a deformation of containers due to explosion, containers were blown up by explosives, and taken by high speed camera. Experimental results showed that the supporting strings are available for suppressing deformation.
\end{abstract}

\section{Introduction}

It is expected that power modules will be popularized due to the influence of smart grid and soon, but when dielectric breakdown occurs when high voltage exceeding breakdown voltage is applied, explosion occurs due to high heat of chip of component parts have been confirmed. Besides, there are many things that have a risk of explosion around us, such as lithium ion batteries used in smart phones, headphones, etc. and spray cans used in paints, insecticides, etc. Because of the possibility of damage to the surrounding people and things due to the explosion and the fragments of the explosive component parts, it is essential to establish a method to protect the surroundings from explosion and fragments in order to operate safely.

As a method of protecting the surroundings from explosion and fragments, it is conceivable to develop a container that has strength against explosion and penetration. Strength against shock and high speed penetration of fragments is required for the material of the container. And also, in the case of lithium ion batteries or power modules, containers should be lightweight and compact to incorporate into the equipment in which they are used. As a material of container, UHMW which satisfies the above properties was used for container. Also, UHMW was reinforced by Dyneema string or Zylon string protecting from being broken due to an increase in strain. In order to investigate the influence of penetration of fragments, explosives were loaded in metal pipes and placed inside the container and blown up. High speed deformation due to explosion was observed by high speed camera. It was also investigated whether Dyneema and Zylon can be used as the material of the reinforcing layer. Experiments were conducted in Shock Wave Laboratory, Kumamoto University, Japan.

\section{Explosion experiment of UHMW1}

The deformation of UHMW due to explosion was observed. Sizes of UHMW used in the experiment and a schematic diagram of the experimental device are shown in Fig.1, and the 
device after assembly is shown in Fig.2. To observe the deformation of UHMW, took videos from the radial direction by using a high-speed video camera (PhantomV7.3). Table.1 shows experimental conditions. Detonating fuse (DF), which contains $9.5 \sim 11.5 \mathrm{~g} / \mathrm{m}$ pentaerythritol tetranitrate (PETN), was used as an explosive. In order to confirm whether Dyneema string (3.0 $\mathrm{mm}$ diameter) is available as a reinforcing material, container was exploded while changing the number of reinforcing layers. Also, to confirm the influence of the fragments, DF was installed in a brass pipe and exploded. Next, in order to observe the influence of the deformation due to the change of the explosive amount, explosives was changed to PETN, loaded in an explosive pipe and detonated with an electric detonator. Also, in order to observe the influence by the fragments, we made experiments while changing the material of the explosivepipe.
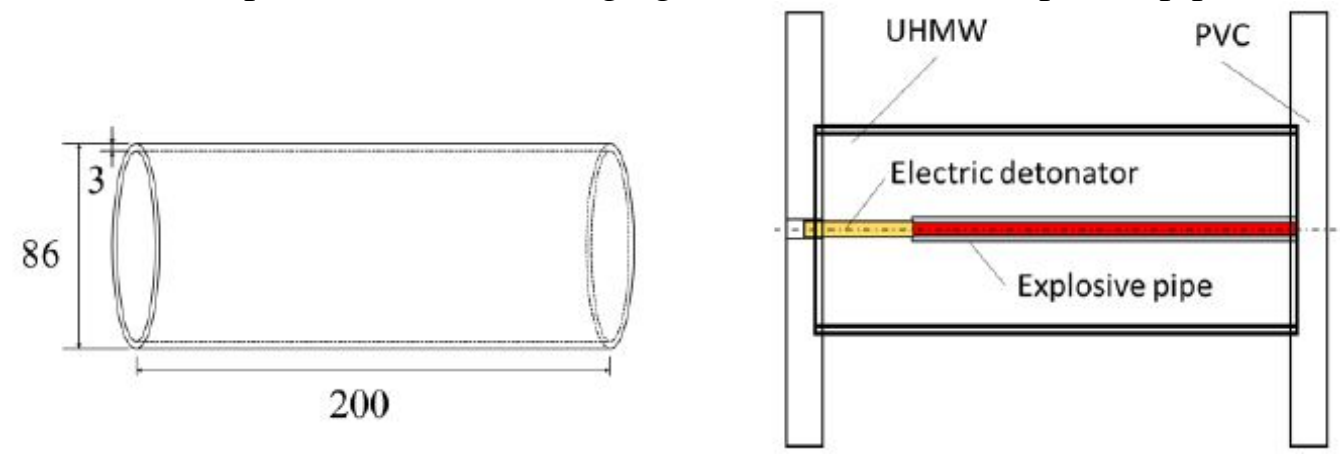

Fig.1 Sizes of UHMW, Schematic diagram of the experimental device

Table.1 Experimental conditions

\begin{tabular}{|c|c|c|c|c|c|c|}
\hline & Explosive & $\begin{array}{c}\text { Reinforcing } \\
\text { layers }\end{array}$ & Brass pipe & $\begin{array}{l}\text { Length of } \\
\text { DF [mm] }\end{array}$ & \multicolumn{2}{|c|}{ Amount of explosive [g] } \\
\hline No.1 & $\mathrm{DF}$ & 0 & None & 160 & \multicolumn{2}{|c|}{1.68} \\
\hline No. 2 & $\mathrm{DF}$ & 1 & None & 160 & \multicolumn{2}{|c|}{1.68} \\
\hline No.3 & DF & 2 & Used & 160 & \multicolumn{2}{|c|}{1.68} \\
\hline No.4 & $\mathrm{DF}$ & 2 & None & 190 & \multicolumn{2}{|c|}{1.995} \\
\hline & Explosive & $\begin{array}{c}\text { Reinforcing } \\
\text { layers }\end{array}$ & $\begin{array}{c}\text { Explosive } \\
\text { pipe }\end{array}$ & $\begin{array}{c}\text { Length of } \\
\text { explosive part } \\
\text { [mm] }\end{array}$ & $\begin{array}{c}\text { Amount of } \\
\text { explosive [g] }\end{array}$ & $\begin{array}{c}\text { Inner diameter } \\
\text { of cylinder } \\
\text { [mm] }\end{array}$ \\
\hline No.5 & PETN & 2 & Straw & 160 & 3.466 & 5.50 \\
\hline No.6 & PETN & 2 & Aluminum & 150 & 9.739 & 10.00 \\
\hline No.7 & PETN & 1 & Copper & 160 & 3.885 & 6.00 \\
\hline No.8 & PETN & 2 & Copper & 160 & 6.269 & 7.52 \\
\hline
\end{tabular}



Fig.2 Experimental device after assembly 


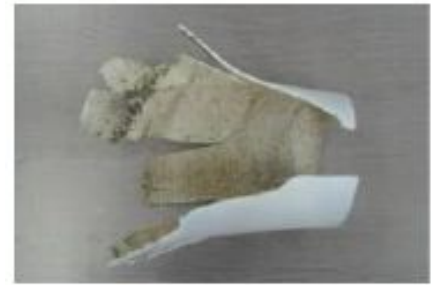

No.1



No.6

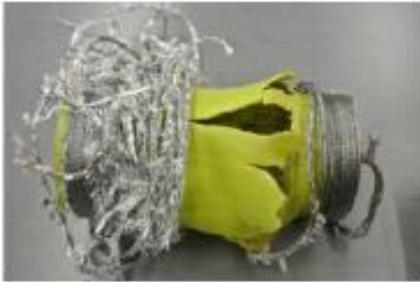

No.7

Fig3. Samples after exploded

Samples after exploded are shown in Fig.3. In the case of using DF, Only No.1 that was not reinforced by Dyneema was broken, and No.2, 3 and 4 that were reinforced showed no significant change. From these, it can be said that Dyneema is available for reinforcement material. In the case of using PETN, No.6 was broken. In No. 6, due to the increase in the amount of explosives, Dyneema was partially cut, and a part of UHMW expanded but wasn't broken. In the case of No.8, there were traces that the fragments penetrated at the end part, but wasn’t broken. In No.5, no significant change was observed.

\section{Explosion experiment of UHMW2}

It was observed how the difference of material of explosive pipe affects the deformation of UHMW. ABS and copper pipes are used as a material of explosive pipe, conducted experiment while changing the amount of explosive. The material of the side plate was changed to SS400 in order to prevent the decrease in pressure due to the destruction. The experimental procedure is the same as in Chapter 2. A schematic diagram of the device is shown in Fig.4, and the device after assembly is shown in Fig.5. To observe the deformation of UHMW, took videos from the radial direction by Phantom V7.3. The experimental conditions are shown in Table.2. The reinforcement of Dyneema is 2 layers, and the length of explosive pipe is $160 \mathrm{~mm}$. In order to compare the influence of the differences in the material of the fragments, experiments using ABS for explosive pipe were conducted twice, and experiments using a copper pipe were conducted twice.

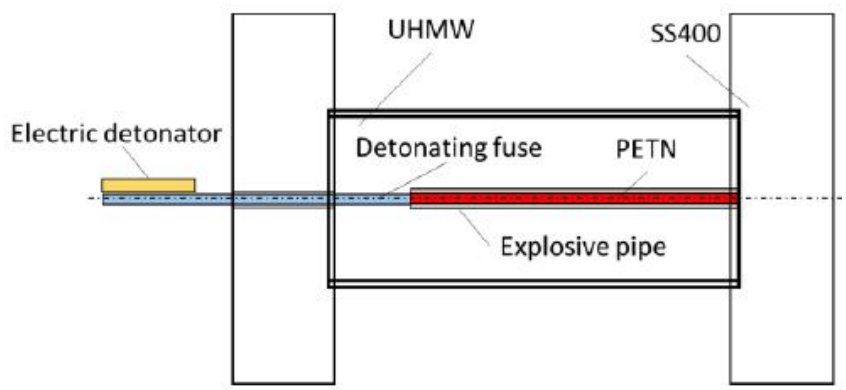

Fig.4 Schematic diagram of the device,

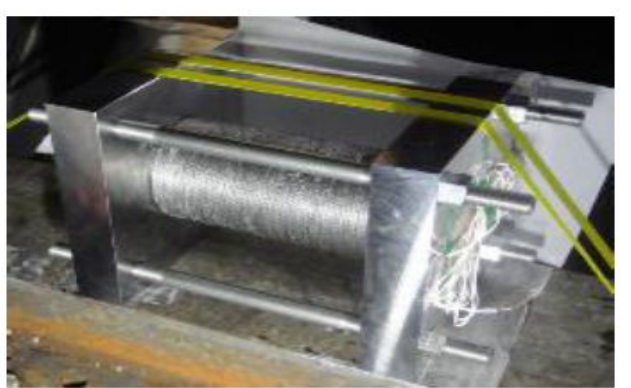

Fig.5 Experimental device after assembly,

Samples after exploded are shown in Fig.6. No.9 was not broken, but No.4, the amount of explosive was almost the same as No.9, was broken. No.10, which used the largest amount of explosives, was also broken. In the case of No.11 that used copper pipe as the material of explosive pipe and DF as explosive, it wasn't broken. Because the container was cracked from the part where the Dyneema was cut, it is thought that penetration of fragments greatly affects the destruction of the container. 
Table.2 Experimental conditions

\begin{tabular}{|l|c|c|c|c|c|}
\hline \multirow{2}{*}{} & \multicolumn{3}{|c|}{ Explosive pipe } & Explosive & $\begin{array}{c}\text { Amount of } \\
\text { Explosive [g] }\end{array}$ \\
\cline { 2 - 5 } & Material & Inner dia [mm] & Thickness [mm] & & 6.9 \\
\hline No.9 & ABS & 8 & t2 & PETN & 13.5 \\
\hline No.10 & ABS & 11 & t2 & PETN & 2.0 \\
\hline No.11 & Copper & 2.5 & t1 & DF & 7.0 \\
\hline No.12 & Copper & 8 & t1 & PETN & \\
\hline
\end{tabular}

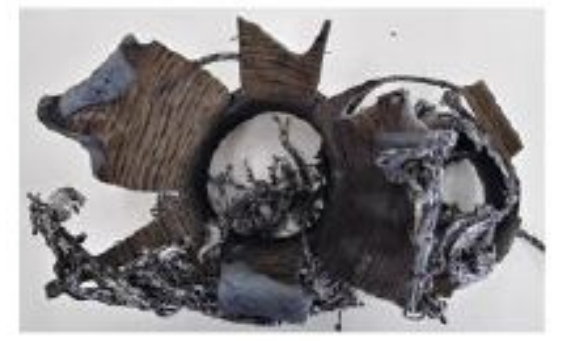

No.10

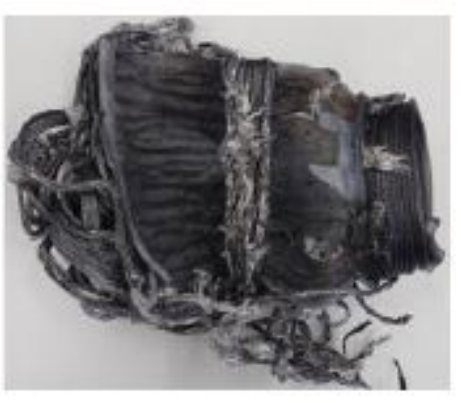

No.12

Fig.6 Samples after exploded

\section{Study on reinforcement material}

The influence of difference of reinforcement materials on the deformation of UHMW was compared. Besides Dyneema which has excellent tensile strength, ZylonX and Zylonknot, which have higher tensile strength than Dyneema, were used. The diameter of the reinforcement string is $3 \mathrm{~mm}$ for Dyneema, $1.9 \mathrm{~mm}$ for ZylonX, and $2.6 \mathrm{~mm}$ for Zylonknot. The size of UHMW used in the experiment and the schematic of the experimental device are shown in Fig.7. Copper was used for the material of explosive pipe, and the influence of fragments was observed. The experimental procedure is the same as in Chapter 2. To observe the deformation of UHMW, took videos from the radial direction by Phantom V 7.3. The experimental conditions are shown in Table.3, and physical properties of the reinforcing string material are shown in the Table.4, [1], [2]. The reinforcement for all samples is one layer.



Fig.7 Sizes of UHMW, Schematic diagram of the experimental device 
Table.3 Experimental conditions

\begin{tabular}{|c|c|c|c|c|c|}
\hline & \multirow{2}{*}{$\begin{array}{l}\text { UHMW size } \\
{[\mathrm{mm}]}\end{array}$} & \multicolumn{2}{|c|}{ Reinforcing string } & \multirow{2}{*}{$\begin{array}{l}\text { Copper pipe } \\
\text { size }[\mathrm{mm}]\end{array}$} & \multirow{2}{*}{$\begin{array}{l}\text { Amount of } \\
\text { Explosive [g] }\end{array}$} \\
\hline & & Material & Diameter [mm] & & \\
\hline No.13 & \multirow{2}{*}{$\begin{array}{l}\text { Inner dia } 104.6 \\
\text { Outer dia } 110.6 \\
\text { width } 40.5\end{array}$} & Dyneema & 3 & \multirow{2}{*}{$\begin{array}{l}\text { Inner dia } 6 \\
\text { Thickness } 1 \\
\text { width } 30.5\end{array}$} & 0.736 \\
\hline No.14 & & ZylonX & 1.9 & & 0.734 \\
\hline No.15 & \multirow{4}{*}{$\begin{array}{l}\text { Inner dia } 104.6 \\
\text { Outer dia } 110.6 \\
\text { width } 46.5\end{array}$} & Zylonknot & 2.6 & \multirow{2}{*}{$\begin{array}{l}\text { Inner dia } 6 \\
\text { Thickness } 1 \\
\text { width } 36.5\end{array}$} & 0.860 \\
\hline No.16 & & Dyneema & 3 & & 0.848 \\
\hline No.17 & & Zylonknot & 2.6 & \multirow{2}{*}{$\begin{array}{l}\text { Inner dia } 7.52 \\
\text { Thickness } 1 \\
\text { width } 36.5\end{array}$} & 1.397 \\
\hline No.18 & & Dyneema & 3 & & 1.408 \\
\hline
\end{tabular}

Table.4 Physical properties of the reinforcing string material

\begin{tabular}{|c|c|c|c|}
\hline & $\begin{array}{c}\text { Tensile strength } \\
{[\mathrm{GPa}]}\end{array}$ & $\begin{array}{c}\text { Tensile modulus } \\
{[\mathrm{GPa}]}\end{array}$ & $\begin{array}{c}\text { Bulk modulus } \\
{[\mathrm{GPa}]}\end{array}$ \\
\hline Dyneema & 2.5 & 123 & 68.3 \\
\hline ZylonX & 4.2 & 180 & 100 \\
\hline Zylonknot & 4.0 & 120 & 66.7 \\
\hline
\end{tabular}

Samples after exploded are shown in Fig.10. No.8 reinforced with ZylonX was broken, but UHMW was not broken in No.5, No.7 and No.8 which were exploded at almost the same amount of explosive, and reinforcing layer was cut at the part where the copper fragments was penetrated. No.17 and No.18 which used a large amount of explosives were also broken.

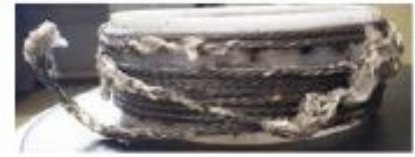

No.13

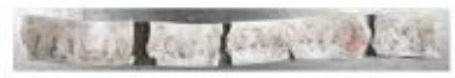

No. 14

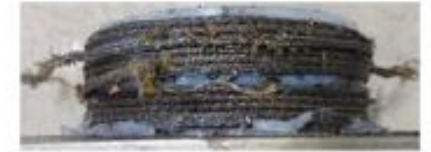

No. 15

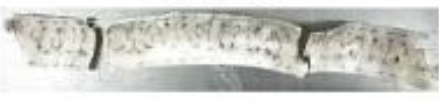

No.17

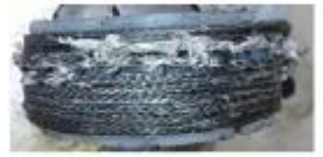

No.16

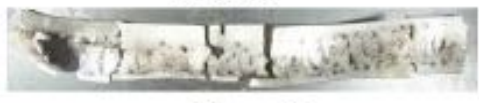

No.18

Fig.8 Samples after exploded

\section{Influence of metal fragments on deformation}

The influence of the amount and size of metal fragments on the deformation of UHMW was investigated. UHMW sizes and experimental procedures are the same as in Chapter 2. A schematic diagram of the experimental device is shown in Fig.9. The length of explosive pipe is $194 \mathrm{~mm}$ to make the fragments collide with UHMW uniformly. The explosive pipe was made of two layers, copper and ABS. The amount and size of fragments were adjusted by the diameter of the copper pipe, and the amount of explosives was adjusted by the diameter of the ABS pipe. The experimental conditions are shown in Table.5. Dyneema was used as the material of the reinforcing layer. The reinforcement is two layers.

A graph measuring the time change of the radius of the container due to the explosion is shown in Fig.10. Measured the central part of the container every $16 \mu$ s, which is the frame interval of a high-speed camera. All samples were not broken, and containers were repeatedly expanding and contracting. As shown in Fig.7, No.17 which used a large copper pipe repeats expansion and contraction with large displacement. It is thought that larger fragments are generated if a large copper pipe is used, and causes a large deformation of the container. 


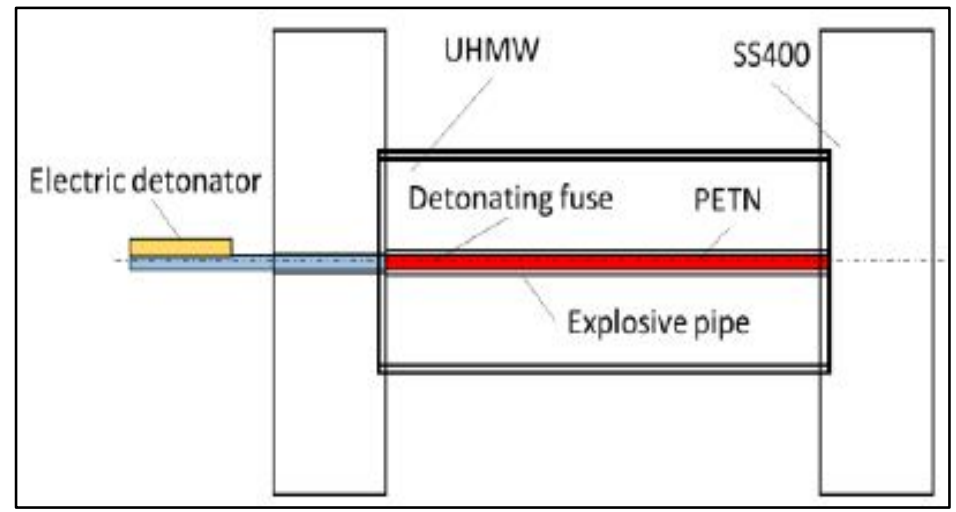

Fig.9 Schematic diagram of the experimental device

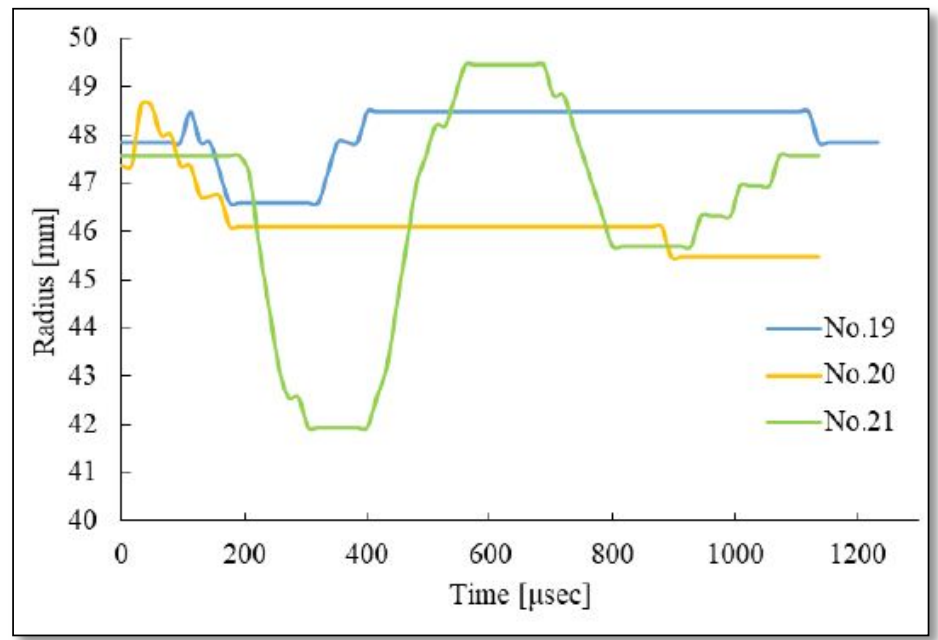

Fig.10 Time change of radius of container

Table.5 Experimental condition

\begin{tabular}{|l|l|l|l|l|l|}
\hline & \multicolumn{2}{|l|}{ Explosive pipe ABS } & \multicolumn{2}{l|}{ Explosive pipe Copper } & Amount of \\
\cline { 2 - 5 } & $\begin{array}{l}\text { Inner dia } \\
{[\mathrm{mm}]}\end{array}$ & $\begin{array}{l}\text { Thickness } \\
{[\mathrm{mm}]}\end{array}$ & $\begin{array}{l}\text { Inner dia } \\
{[\mathrm{mm}]}\end{array}$ & $\begin{array}{l}\text { Thickness } \\
{[\mathrm{mm}]}\end{array}$ & explosive [g] \\
\hline No.19 & 4 & $\mathrm{t} 1$ & 6 & $\mathrm{t} 1$ & 2.156 \\
\hline No.20 & 6 & $\mathrm{t} 0.7$ & 7.52 & $\mathrm{t} 1$ & 4.354 \\
\hline No.21 & 6 & $\mathrm{t} 1$ & 15 & $\mathrm{t} 1.5$ & 4.217 \\
\hline
\end{tabular}

\section{Conclusions}

It was confirmed that it is available as enforcing material for Zylon and Dyneema. It was found that the penetration of fragments has a great influence on the deformation of the container. It was found that the rigidity of the reinforcing layer material is related to the destruction of the container.

\section{References}

[1] TOYOBO.(n.d.). Product information of IZANAS, Rerived March 16, 2019,from http://www.toyobo.co.jp/seihin/dn/izanas/iz_product.html

[2] TOYOBO.(n.d.). Product information of ZYLON, Rerived March 16, 2019, from http://www.toyobo.co.jp/seihin/kc/pbo/zylon_features.html 